\section{Determination of the convective heat transfer coefficient}

Spierings ThAM, Bosman F, Peters MCRB, Plasschaert AJM. Determination of the convective heat transfer coefficient. Dent Mater 1987: 3: 161-164.

\begin{abstract}
The value of the convective heat transfer coefficient (htc) is determined under different loading conditions by using a computer aided method. The thermal load has been applied mathematically as well as experimentally to the coronal surface of an axisymmetric tooth model. To verify the assumptions made for the mathematical tooth model, the results predicted with this model were compared with those of an experiment using mercury as the tooth surrounding medium. For all the other thermal loading conditions the medium water has been used. During the experiments the temperatures were recorded at one point within the resin model. The temperatures within the theoretical model have been calculated according to the FEA. Comparison of the calculated data with the experimental recordings led firstly to a verification of the theoretical model, and secondly to a htc-value of $5.10^{4} \mathrm{~J} / \mathrm{m}^{2} \mathrm{~s}^{\circ} \mathrm{C}$. From the findings of this study it can be concluded that this value of the htc satisfies for the experimental conditions used. However, additional in vivo experiments will be needed to verity the htc-value during the consumption of liquids.
\end{abstract}

D. Spierings', F. Bosman², T. Peters ${ }^{1}$, F. Plasschaert ${ }^{1}$

${ }^{1}$ Department of Cariology and Endodontology, University of Nijmegen, ${ }^{2}$ Department of Oral Patho-physiology, University of Utrecht, The Netherlands

Keywords: heat transfer, tooth, temperature, mathematics

Th. A. M. Spierings, Department of Cariology and Endodontology, University of Nijmegen, $\mathrm{PO}$ Box 9101, 6500 HB Nijmegen, The Netherlands

Received April 21, 1986; accepted June 12, 1986.
The convective heat transfer coefficient turned out to be an essential parameter in a study on temperature distribution in restored human teeth using a mathematical model (1). In convective heat transfer processes, the heat transfer coefficient (htc) represents the quantity of thermal energy transferred in a unit time at a fluid-solid interface of a unit area having a unit temperature difference. Its value is a complex function of the flow rate of the fluid, the fluids physical parameters, and the interface geometry of the solid into which or from which the thermal energy is transferred.

In the oral cavity most values fell in the $3.400-11.400 \mathrm{~J} / \mathrm{m}^{2} \mathrm{~s}^{\circ} \mathrm{C}$ range as published by Boehm (3). Enlarging the value of the htc results in an increase in temperature change of the underlying structures due to the faster energy transfer. In general, the heat transfer of a beverage will be larger in comparison with solid food of the same temperature (2). So, the temperature changes in teeth are larger from drinking a hot beverage than eating hot solid food of the same temperature. Since we are interested in the worst case, the thermal load in this study will be limited to fluid applications.

Jacobs (2) investigated the heat transfer coefficient during application of a constant flow rate of the fluid. In the oral cavity, however, the flow rate is not constant drinking a liquid. Moreover, the outer surface of the dentition is not necessarily uniformly wetted. Thus the value of the htc during the heat transfer may vary over the outer surface of the coronal part of the dentition. Determination of the htc-value for such a complex process might yield a mean value, which depends on the physical factors involved. In recent studies on temperature distribution in restored teeth $(1,4)$, the value of htc given by Jacobs (2) has been used. However, comparison of our results with the in vivo measurements of Tibbetts (5) indicates that a larger value of htc might be more realistic (4). Since the value of the htc is an important parameter in studies concerning heat transport, and since only limited data on the value of htc could be derived from literature, it is the purpose of this study to determine its value under different loading conditions.

The complexity of and variation in human tooth structure make natural teeth unsuitable for standardized experiments. This points to the needs to used a simplified axisymmetric tooth model. Since this heat transfer process is complex in nature, it is rather difficult to determine the value of the htc in the laboratory alone. Consequently, a computer-aided method was used. The different thermal loading situations were exerted in theoretical simulation models as well as in laboratory experiments. Combining the calculated data of the theroretical model with the results of the corresponding experiments will yield the value of htc for the given conditions.

\section{Material and methods}

A numerical, as well as a corresponding 


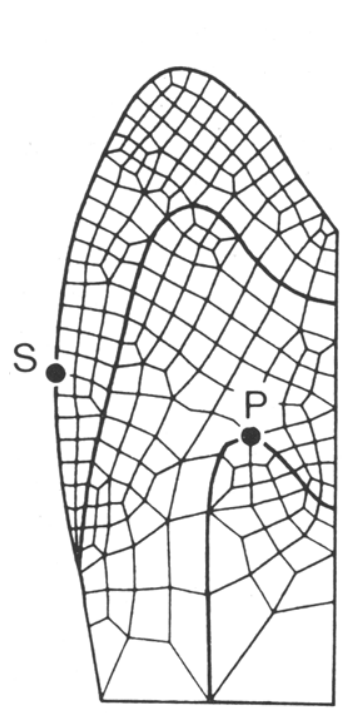

$-A-$

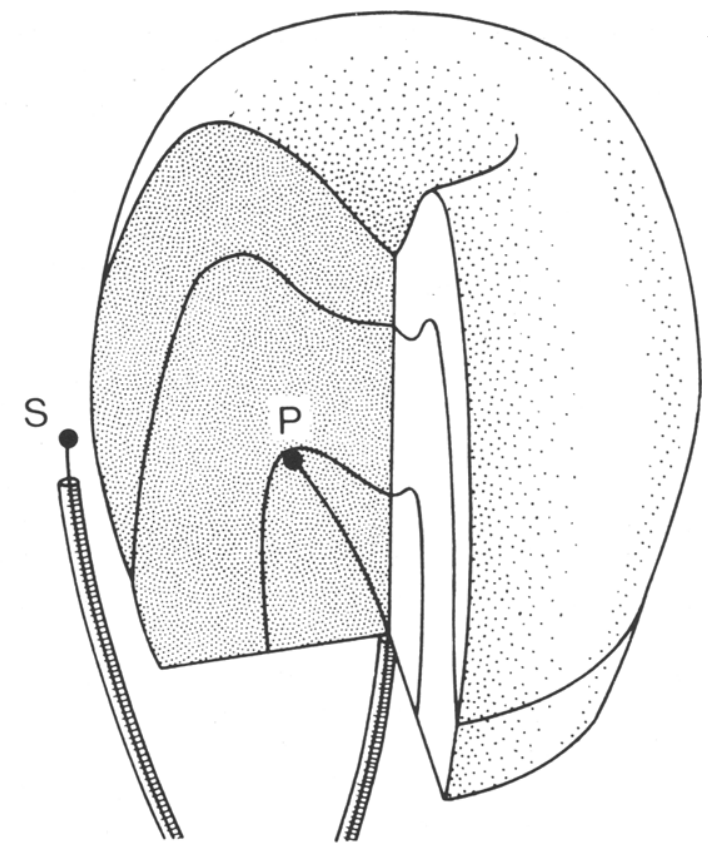

- B-

Fig. 1. (A) cross-section of the axisymmetric model, including the element mesh; and the nodes $S$ and $P$ corresponding to the 2 thermocouples $S$ and $P$ of the replica $(B)$.

experimental, method has been used to determine the value of the htc for water running along the coronal surface of an axisymanetric tooth. The geometry of each theoretical and experimental, axisymmetric tooth model was based on the buccopalatal contours of a maxillary first premolar tooth, as derived from an X-ray (Fig. 1). These models were composed of one material, i.e. epoxy resin.

When one is drinking a liquid, only the coronal part of the tooth is involved. This situation comprises several physical parameters. It also introduces the question of whether the apical side must be kept at a constant temperature or has to be loaded similarly to the coronal part. Thus, this question has to be answered before experiments are carried out with water as the surrounding liquid, and before a comparison can be made to the corresponding theoretical model. Therefore, one experiment is performed with mercury as the medium, which has compared to drinks effectively an infinite large value of htc. This implies that there is essentially no temperature gradient at the outer surface of the model (6).

To determine the value of the htc, the following loading conditions were simulated: (A) a constant temperature of $0^{\circ} \mathrm{C}$ applied at the coronal surface of the model for at least $60 \mathrm{~s}$, using mercury. At the apical side of the model, the temperature is constant, i.e., equal to the initial temperature of the model; (B) the same as A, however, stagnant water is used instead of mercury; (C) the same as B, however, the load is applied for $10 \mathrm{~s}$, and then the model is returned into an ambient condition of $30^{\circ} \mathrm{C}$ for the remainder of time $(50 \mathrm{~s})$; and (D) a draught of $30 \mathrm{ml}$ water at $0^{\circ} \mathrm{C}$ is simulated to determine a possible influence of running instead of stagnant water on the value of the htc.

For practical reasons, the initial temperature of the model was $30^{\circ} \mathrm{C}$. In the theoretical and experimental models, the temperatures caused by the various thermal loads have been calculated or recorded at one point. This point corresponds with the top of the pulpo-dentinal junction (Fig. 1). The comparison of the theoretical data with the experimental results could lead to a verification of the theoretical model to the determination of the htc-value.

\section{Theoretical approach}

The calculations were carried out using to the finite element method. Therefore, an element mesh was made for the axisymmetric tooth model using quadratic ring elements with a bi-qaudratic temperature field. The total number of elements and nodes was 238 and 769 , respectively (Fig. 1).

The material properties of the epoxy resin used were assumed to be isotropic, homogeneous, and temperature independent. Its assigned value of thermal diffusivity was $1.4010^{-7} \mathrm{~m}^{2} / \mathrm{s}(7)$.

The heat input at the coronal surface of the model depends on the temperature difference across the tooth-oral cavity interface, the ambient temperature and the htc. In formula:

$\mathrm{q}_{\mathrm{n}}=\alpha\left(\mathrm{T}_{\mathrm{b}}-\mathrm{T}_{\infty}\right)$

$\left(\mathrm{q}_{\mathrm{n}}=\right.$ heat flux perpendiculaqr to surface; $\alpha=$ heat transfer coefficient (htc); $\mathrm{T}_{\mathrm{b}}=$ local boundary temperature; $\mathrm{T}_{\infty}=$ ambient temperature). At the apical side, the temperature difference between the environment and tooth surface is assumed to be zero. In view of the assumptions the boundary conditions are linear in nature.

As a result of the axisymmetry, there is no heat transport in a tangential direction (i.e., at right angles to each cross-section). For the replica is composed of one material, the heat transfer in the tooth model is based on the heat balance law and Fourier's heat conduction law.

To complete the mathematical model it was assumed that there is no heat radiation from the tooth model.

Using mercury as the medium (load A), the htc was given the value of $5.10^{12}$ $\mathrm{J} / \mathrm{m}^{2} \mathrm{~s}^{\circ} \mathrm{C}$, resulted in a tooth surface temperature equal to the temperature of the mercury. Calculations were made and the results were verified with those from the corresponding laboratory experiments.

After the verification of the modeling chosen, the value of htc will be fitted to match the calculated data with the results of the experiments, following from the given loading conditions $\mathrm{B}$ and $\mathrm{C}$ using water as the medium.

The value of the htc pertaining to load B, using water as the medium, was used to simulate load D. One draught of liquid was simulated as a function of time by the error function erf $(0.453 /$ $\sqrt{ } \mathrm{t})(8)$. The results of the modeling chosen were compared with the experimental data.

\section{Experimental approach}

To prepare a replica of the axisymmetric tooth model, an epoxy resin* was 
Table 1. Direction coefficient of $\ln \left(\mathrm{T}(\mathrm{t}) / \mathrm{T}_{0}\right)$ and the corresponding heat transfer coefficient (htc) as result of the thermal loads $\mathrm{A} / \mathrm{B}$.

\begin{tabular}{lcccccc}
\hline medium & \multicolumn{2}{c}{ thermal load $\left({ }^{\circ} \mathrm{C}\right)$} & & \multicolumn{2}{c}{$\begin{array}{c}\text { direction coefficient } \\
\ln \left(\mathrm{T}(\mathrm{t}) / \mathrm{T}_{\mathrm{o}}\right)\end{array}$} & $\begin{array}{c}\text { Value } \\
\text { of } \\
\mathrm{htc}\end{array}$ \\
\cline { 2 - 3 } & theory & experiment & & theory & experiment & $\mathrm{J} / \mathrm{m}^{2} \mathrm{~s}^{\circ} \mathrm{C}$ \\
\hline A: mercury & 0.0 & $0.7 \pm 0.2$ & & -0.023 & $-0.021 \pm 0.001$ & $\infty$ \\
B: water & 1.5 & $1.6 \pm 0.4$ & & -0.021 & $-0.019 \pm 0.001$ & $5.10^{4}$ \\
\hline
\end{tabular}

$T(t)$ and $T_{0}$ are temperatures at time $t$ and $t=0$, respectively. poured in a silicone rubber $^{\dagger}$ mold in which the hot junction of a copper-constantan thermocouple was located (Fig. 1) at the point corresponding to $P$ in the theoretical model. The resin was mixed according to the manufacturers' instructions.

For initial heating the replica was stored for at least $60 \mathrm{~min}$ in a hot-air stove at a constant temperature of $30^{\circ} \mathrm{C}$.

A mercury bath was used to verify the theoretical model. The mercury was maintained at a loading temperature of $0^{\circ} \mathrm{C}$ by a jacket of ice water. The replica was immersed into the mercury bath as specified in loading situation $A$.

The temperature of the water bath was maintained constant at $0^{\circ} \mathrm{C}$, using ice-water. The experiments without running water (loads $B$ and $C$ ) were carried out like the one with mercury; $30 \mathrm{ml}$ of ice-water was used to simulate loading condition D (a draught of liquid). The water was applied on top of the replica.

During the various thermal loadings, the change of the ambient temperature and the temperature change within the replica were recorded using a copperconstantan thermocouple. The reference junctions of the thermocouples were kept in ice-water. The thermocouple output was amplified ${ }^{\ddagger}$ and recorded versus time using a tape recorder $^{\S}$. All experiments were repeated 5 times.

For comparison of the theoretical and experimental data of the loading conditions $\mathrm{A}$ and $\mathrm{B}$, all temperaturetime curves were plotted for $\ln \left(T(t) / T_{0}\right)$ as a function of time, where $T$ is the measured temperature at time $t$, and $T_{0}$

* Araldite D + Harder HY 956 (20\%), Ciba-Geigy, Basel, Switserland.

† Wacker Silicone: RTV-M45/ + Harder T40, Wacker-chemie, GMSH-München, Germany.

* Differential Amplifier 26A-2601 R2601

Mainframe, Tektronix, Inc., Beaverton, Oregon, 97005, USA.

3968 Instrumentation Recorder, Hewlet Packard, Palo Alto, Ca 94304 USA. is the initial temperature of the replica. Under the given loading conditions, the theoretical data had to correspond with the given results of the laboratory experiments.

During the transport of the replica from air-stove to thermal loading place, its initial temperature will be lower than in the theoretical model. Therefore, all data obtained from the loading conditions $\mathrm{C}$ and $\mathrm{D}$ were normalized first, by dividing the calculated or recorded temperatures by the initial temperature of the model.

\section{Results}

The mean initial temperature of the replica was $29.3 \pm 0.5^{\circ} \mathrm{C}$. for each loading condition ( $A$ and $B$ ), the chosen thermal load value (theory) as well as the recorded average loading temperature (experimental) is given in Table 1. The modeling chosen in loading condition A fits the experimental results using mercury. Thus for loading condition $B$, using water as medium instead of mercury, the given value of htc appeared to be the best value of the parameter fitting the experimental data. Increasing this value had no significant influence on the calculated data at point $P$.

In Fig. 2, the normalized temperatures at point $\mathbf{P}$ for load $\mathbf{C}$ are plotted as a function of time. The calculated temperatures at node $\mathrm{P}$ using a htc of $5.10^{4} \mathrm{~J} / \mathrm{m}^{2} \mathrm{~s}^{\circ} \mathrm{C}$ are in agreement with the data obtained experimentally, whereas a smaller value of htc appeared to have a negative influence on the magnitude of the calculated data. Enlarging the htc-value had still no significant influence on the temperatures at node $P$. The thermal load caused at $\mathrm{P}$ a minimum temperature of $24.8^{\circ} \mathrm{C}$ in the theoretical model (i.e., htc is $5.10^{4}$ ) and $24.1 \pm 0.4^{\circ} \mathrm{C}$ within the replica.

The calculated and recorded normalized temperatures within the model (P) and at the surface of the model (S) as a result of load D, are depicted in Fig. 3. Although point $S$ at the surface of the theoretical model does not correspond exactly with the localization of the nearby thermocouple, the calculated and recorded data show close agreement. For point $\mathrm{P}$, however, the initial stages of the curves show a difference in temperature change.

\section{Discussion}

In this study, only one thermocouple was situated in the replica. If more than one measuring site would have been

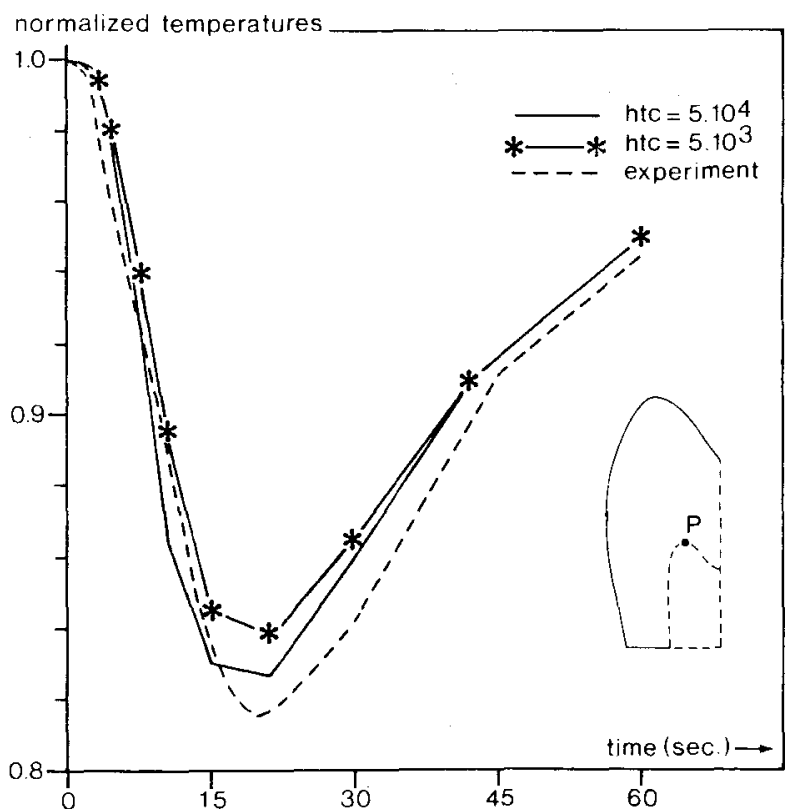

Fig. 2. The normalized temperatures for load $\mathrm{C}$ at point $\mathrm{P}$ in the theoretical model and in the replica, having a heat transfer coefficient (htc) of $5.10^{4}$ and $5.10^{3} \mathrm{~J} / \mathrm{m}^{2} \mathrm{~s}^{\circ} \mathrm{C}$ during a period of $60 \mathrm{~s}$. 


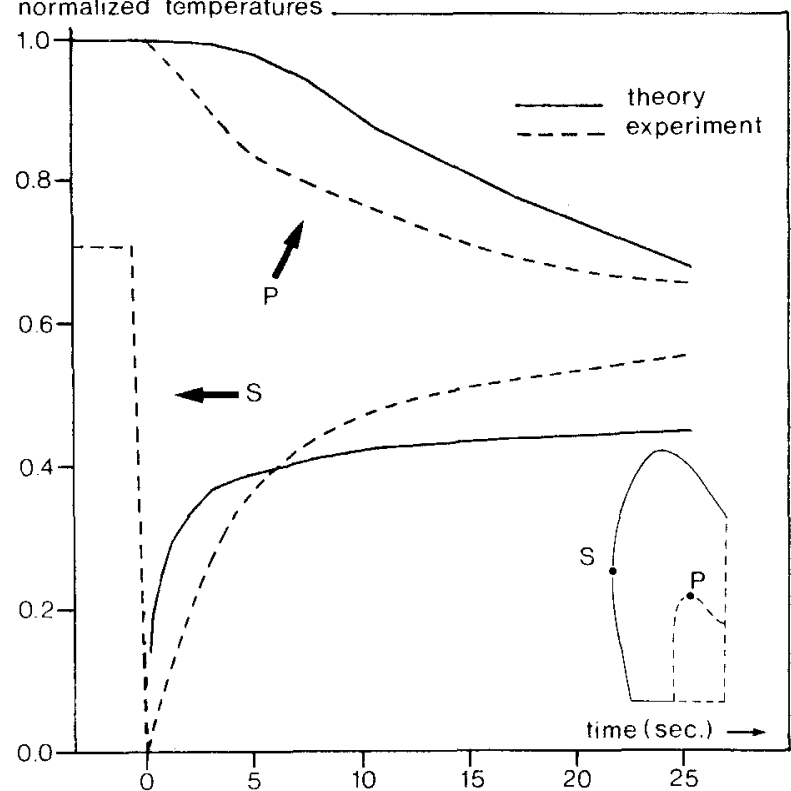

Fig. 3. The normalized temperatures for load $\mathrm{D}$ at the points $\mathrm{S}$ and $\mathrm{P}$ in respectively the theoretical model and the replica during a period of $25 \mathrm{~s}$ after initiation of the heat flux. However, the thermocouple (S) was located near point $S$ not in it.

present, more information could have been obtained about the temperatures at these sites and about the temperature gradient between these points. However, the use of more than one thermocouple in such a small model was not possible without influencing the temperature field. The use of only one thermocouple is therefore limiting the conclusions.

With respect to the temperatures at point $P$, all calculated results using FEA correspond with the experimental data using the given value of htc. However, enlarging the htc did not result in other calculated temperatures at $\mathrm{P}$, whereas the temperature gradient to the surface increased. On the other hand, reduction of this value caused smaller temperature changes within the entire mathematical model (Fig. 2). Thus, the value of htc obtained represents the lower bound for the given conditions. If the thermocouple was located near the surface, it could be disputed whether the results could inform us better about this parameter, specifically if the load is not uniformly applied to the coronal part of the model. Further research will be necessary to determine the 'mean' value of htc during the heat transfer process of drinking a beverage, leading to a more accurate figure for the temperature level at the surface of a tooth.
The temperature differences between the curves for node $\mathrm{P}$ as shown in Fig. 3 can be attributed to the influence of running water on the value of htc, indicating that this value should be higher. In this study, however, the application of the liquid on top of the replica caused a uniform-wetted surface, whereas in vivo the wetting degree will not be uniform at the coronal part of teeth when drinking a beverage. Moreover, enlarging the value of htc did not have any influence on the temperatures at node $P$. Therefore, it is recommended that loading situation $\mathrm{D}$ be repeated in an in vivo experiment. These results could then be compared to the data obtained from the theoretical model simulating this loading condition. From those findings, it might be concluded whether the value of htc, i.e., $5.10^{4}$, represents the 'mean' value.

The values of htc for liquids determined by Jacobs (2) are lower than the value presented in this study. In his study, the fluid was poured over a small cylinder used as measuring object. It is in question whether in that situation the volumetric flow rate of $6.0 \mathrm{~cm}^{3} / \mathrm{s} \mathrm{re}$ sulted in a similar situation along the surface of the dentition while drinking a beverage. A smooth surface copper cylinder was used, whereas the tooth replica used in our study had a rougher surface. This may cause a different tur- bulent flow and therefore higher heat transfer for the rougher surfaced model resulting in an effectively larger wetted area for the courser model, which further enhances heat transfer even under no-flow conditions. Moreover, the flow velocity of liquids passing the oral cavity is not known from literature. These factors could be responsible for the reported differences. The large value of htc appears to correspond with the calculations on temperature distribution within restored teeth as reported before (4).

From the findings of our study, it can be concluded that (A) the htc-value of $5.10^{4} \mathrm{~J} / \mathrm{m}^{2} \mathrm{~s}^{\circ} \mathrm{C}$ satisfies the experimental conditions used; and (B) additional in vivo experiments will be needed to determine the 'mean' value of the convective heat transfer coefficient during and after the consumption of liquids.

Acknowledgements - This research was part of the research program "Restorations and Restorative Materials".

\section{References}

1. Spierings ThAM, de Vree JHP, Peters MCRB, Plasschaert AJM. The influence of restorative dental materials on heat transmission in human teethh. $J$ Dent Res 1984: 63: 1096-1100.

2. Jacobs HR, Thompson RE, Brown WS. Heat transfer in teeth. $J$ Dent Res 1973 : 52: $248-52$.

3. Boehm RF. Heat transfer in teeth. In: Shitzer A, Eberhart R, eds. Heat transfer in Medicine and Biology. Vol. II. New York: Plenum press, 1985: 307-331.

4. Spierings ThAM, Peters MCRB, Bosman F, Plasschaert AJM. The influence of cavity geometry on heat transmission in restored teeth. $J$ Dent 1986: 14: 47-51.

5. Tibbetts VR, Schnell RJ, Swartz ML, Phillips RW. Thermal diffusion through amalgam and cement bases: comparison of in vitro and in vivo measurements. $J$ Dent Res 1976: 55: 441-51.

6. Carslaw HS, Jaeger JC. Conduction of heat in solids. Oxford: The University Press, 1973: 184-86.

7. Spierings ThAM, Bosman F, Peters MCRB, Plasschaert AJM. Thermal diffusivity of two replica resins. Dent Mater (in press).

8. Spierings ThAM, van der Varst PGT, Peters MCRB, Bosman F, Plasschaert AJM. Thermal fluctuations in the oral cavity during consumption of hot and cool drinks. In: Spierings Th. A. M., ed. Modelling and analysis of thermal loading and heat transport in restored teeth. Thesis: University of Nymegen, 1986: 51-60. 